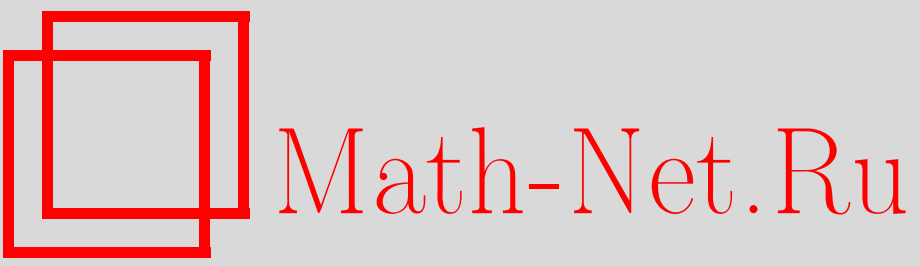

А. А. Кащенко, Динамика одной модели с запаздыванием и большим параметром, Итоги науки и техн. Сер. Соврем. мат. и ее прил. Темат. обз., 2021, том 194, 115-123

DOI: https://doi.org/10.36535/0233-6723-2021-194-115-123

Использование Общероссийского математического портала Math-Net.Ru подразумевает, что вы прочитали и согласны с пользовательским соглашением

http://www . mathnet.ru/rus/agreement

Параметры загрузки:

IP: 54.157 .27 .8

26 апреля 2023 г., 14:29:41

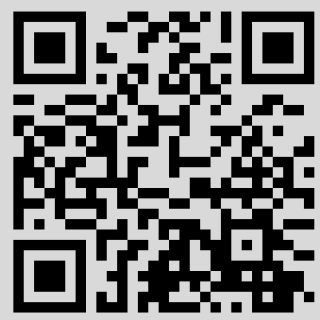




\title{
ДИНАМИКА ОДНОЙ МОДЕЛИ \\ С ЗАПАЗДЫВАНИЕМ И БОЛЬШИМ ПАРАМЕТРОМ
}

\author{
(c) 2021 г. $\quad$ A. A. КАЩЕНКО
}

\begin{abstract}
АннотАция. В работе рассматривается система из двух дифференциальных уравнений с запаздыванием и финитной нелинейностью. С помощью специального асимптотического метода изучаются существование и устойчивость релаксационных периодических решений данной системы в предположении, что положительный множитель перед финитной нелинейностью является достаточно большим. С помощью этого метода задача о поведении решений с начальными условиями из некоторого множества фазового пространства исходной бесконечномерной системы сводится к изучению динамики построенного трехмерного отображения. Доказывается, что грубым циклам отображения соответствуют релаксационные периодические решения исходной системы той же устойчивости. По устойчивым циклам построенного отображения найдены экспоненциально орбитально устойчивые релаксационные циклы исходной системы.
\end{abstract}

Ключевъе слова: асимптотика, релаксационные колебания, периодическое решение, большой параметр, мультистабильность.

\section{DYNAMICS OF ONE MODEL WITH DELAY AND A LARGE PARAMETER}

\section{(c) $2021 \quad$ A. A. KASHCHENKO}

\begin{abstract}
In this paper, we consider a system of two differential equations with delay and a finite nonlinearity. Using a special asymptotic method, we examine the existence and stability of relaxation periodic solutions of the system under the assumption that the positive coefficient of the finite nonlinearity is sufficiently large. This method allows one to reduce the problem on the behavior of solutions whose initial conditions lie in a certain set of the phase space of the original infinitedimensional system to the study of the dynamics of a certain three-dimensional mapping. We prove that rough cycles of this mapping correspond to relaxation periodic solutions of the original system with the same stability. By stable cycles of the mapping constructed, we find exponentially orbitally stable relaxation cycles of the original system.
\end{abstract}

Keywords and phrases: asymptotics, relaxation oscillations, periodic solution, large parameter, multistability.

AMS Subject Classification: 34K13, 34K25

1. Введение. Рассмотрим систему из двух дифференциальных уравнений с запаздыванием

$$
\left\{\begin{array}{l}
\dot{u}_{0}+u_{0}=\lambda F\left(u_{0}(t-T)\right)+\gamma\left(u_{1}-u_{0}\right), \\
\dot{u}_{1}+u_{1}=\lambda F\left(u_{1}(t-T)\right)+\gamma\left(u_{0}-u_{1}\right) .
\end{array}\right.
$$

Работа выполнена при поддержке Российского фонда фундаментальных исследований (проект № 18-29-10055). 
Здесь $u_{i}(i=0,1)$ - скалярные функции, $\lambda, T$ и $\gamma$-положительные параметры. Функция $F$ нелинейная, гладкая, финитная, то есть существует такое положительное число $p$, что

$$
F(u)= \begin{cases}f(u), & |u| \leqslant p \\ 0, & |u|>p .\end{cases}
$$

Данная модель может встречаться как в радиофизике, так и в нейродинамике. Она может описывать динамику двух связанных генераторов с фильтром нижних частот первого порядка и обратной связью (см. $[3,10])$. При дополнительных условиях на функцию $F$ (гарантирующих положительность решения с положительными начальными условиями, наличие импульсных режимов или пачек импульсов) она может быть феноменологической моделью двух связанных нейронов (см. [12]) (о динамике систем с запаздыванием, моделирующих связанные нейроны, см. также $[1,2,5,11])$.

Будем рассматривать более общий случай (как положительные, так и отрицательные начальные условия; функции $f(u)$, при которых система (1) с положительными начальными условиями имеет знакопеременные решения), но в качестве иллюстрации результатов покажем графики режимов, которые можно трактовать как импульсы и пачки импульсов. Предполагаем, что функция $f(u)$ гладкая и удовлетворяет следующим условиям:

$$
\left\{\begin{array}{l}
f(p)=f(-p)=f^{\prime}(p)=f^{\prime}(-p)=0 ; \\
f(u) \neq 0 \text { за исключением конечного числа точек; } \\
\text { если } f\left(u_{*}\right)=0, \text { то } f^{\prime}\left(u_{*}\right) \neq 0 \text { или } f^{\prime \prime}\left(u_{*}\right) \neq 0 .
\end{array}\right.
$$

Ключевое предположение данной работы состоит в том, что положительный параметр $\lambda$ является достаточно большим,

$$
\lambda \gg 1,
$$

а коэффициент связи между уравнениями является достаточно малым:

$$
\gamma=\frac{\gamma_{1}}{\lambda^{\alpha} \ln \lambda}, \quad \gamma_{1}>0, \quad 0<\alpha \leqslant 1 / 2
$$

В [7-9] изучалась нелокальная динамика системы (1) при условиях (2), (4) в предположении $u f(u)>0$ при $0<|u|<p$ при различных порядках малости параметра $\gamma$. Из результатов данных работ следует, что асимптотика найденных релаксационных периодических решений, асимптотика их периода, количество сосуществующих экспоненциально орбитально устойчивых периодических решений зависит от порядка частного параметров $\lambda$ и $\gamma$.

В данной работе изучаются существование и устойчивость релаксационных циклов системы (1) при условиях (2), (3), (4), (5). С помощью специального аналитического метода (см. [4-6]) исследование динамики исходной системы дифференциальных уравнений с запаздыванием и большим параметром удается свести к исследованию динамики более простого трехмерного отображения без больших и малых параметров.

Опишем структуру работы. В разделе 2 изучается асимптотика всех решений системы (1) с начальными условиями из некоторого подмножества $S$ фазового пространства данной системы. В разделе 3 по асимптотике решений конструируется оператор сдвига по траекториям, который множество $S$ отображает в множество «того же типа». Показано, что структура построенного оператора определяется некоторым трехмерным отображением. В разделе 4 формулируется теорема о соответствии грубых циклов построенного отображения релаксационным периодическим решениям исходной системы. В разделе 5 находятся грубые устойчивые циклы построенного отображения, приводятся примеры соответствующих им экспоненциально орбитально устойчивых периодических режимов исходной задачи.

2. Асимптотика решений. Введем обозначения, которые понадобятся нам для определения множества начальных условий для системы (1). Пусть параметр $k$ принимает одно из двух возможных значений: 1 или -1 , параметр $m$ равняется 0 или 1 .

Введем множество начальных условий. Обозначим через $S(m, k, x) \subset C_{[-T, 0]}\left(\mathbb{R}^{2}\right)$ множество непрерывных на $s \in[-T, 0]$ функций $u_{m}(s)$ и $u_{1-m}(s)$, удовлетворяющих следующим условиям: 
(i) при $s$ из полуинтервала $[-T, 0)$ абсолютные величины обеих функций больше, чем $p$ :

$$
\left|u_{m}(s)\right|>p, \quad\left|u_{1-m}(s)\right|>p \quad \text { при } s \in[-T, 0) .
$$

(ii) при $s=0$ функция $u_{m}$ по модулю равна $p$, а функция $u_{1-m}$ по модулю больше, чем $p$ :

$$
u_{m}(0)=k p, \quad u_{1-m}(0)=x p \lambda^{\alpha}, \quad\left(|x| \lambda^{\alpha}>1\right) .
$$

Изучим асимптотику решений с начальными условиями из множества $S(m, k, x)$ при $\lambda \rightarrow+\infty$.

Отметим одно важное обстоятельство. При увеличении времени $t$ для решений системы (1) последовательно чередуются участки двух типов. В первом из них нелинейность не тождественно нулевая, а во втором - нулевая, и тогда функции $u_{0}$ и $u_{1}$ являются решением линейной системы обыкновенных дифференциальных уравнений

$$
\left\{\begin{array}{l}
\dot{u}_{0}+u_{0}=\frac{\gamma_{1}}{\lambda^{\alpha} \ln \lambda}\left(u_{1}-u_{0}\right) \\
\dot{u}_{1}+u_{1}=\frac{\gamma_{1}}{\lambda^{\alpha} \ln \lambda}\left(u_{0}-u_{1}\right) .
\end{array}\right.
$$

Возможность полного асимптотического исследования решений (1) обусловлена тем, что для решений с начальными условиями из множества $S(m, k, x)$ длительность участков первого типа имеет порядок $O(1)$ (при $\lambda \rightarrow+\infty)$, а длительность участков второго типа - асимптотически большая, порядка $O(\ln \lambda)$.

Ниже понадобится формула для решения системы (8). Пусть $\tau$ - некоторый начальный момент времени. Тогда при $t \geqslant \tau$ решение системы (8) имеет вид

$$
\left\{\begin{array}{l}
u_{0}(t)=\frac{1}{2}\left(u_{0}(\tau)+u_{1}(\tau)+\left(u_{0}(\tau)-u_{1}(\tau)\right) \exp \left(-\frac{2 \gamma_{1}(t-\tau)}{\lambda^{\alpha} \ln \lambda}\right)\right) \exp (\tau-t) \\
u_{1}(t)=\frac{1}{2}\left(u_{0}(\tau)+u_{1}(\tau)-\left(u_{0}(\tau)-u_{1}(\tau)\right) \exp \left(-\frac{2 \gamma_{1}(t-\tau)}{\lambda^{\alpha} \ln \lambda}\right)\right) \exp (\tau-t)
\end{array}\right.
$$

Будем строить решения системы (1) пошагово. На первом шаге рассмотрим отрезок $t \in[0, T]$. Из выбора множества начальных условий следует, что при $t \in[0, T]$ функции $F\left(u_{0}(t-T)\right)$ и $F\left(u_{1}(t-T)\right)$ тождественно равны нулю, т.е. система (1) на данном временном промежутке имеет вид (8). Заметим, что при фиксированных значениях параметров $m, k, x$ задача Коши (8), (7) имеет единственное решение. Поэтому на отрезке $t \in[0, T]$ решения системы (1) с начальными условиями из множества $S(m, k, x)$ при фиксированных значениях $m, k, x$ совпадают. Следовательно, они совпадают при всех значениях $t \geqslant 0$.

Из (9) получаем, что рассматриваемое решение исходной системы удовлетворяет асимптотическим равенствам

$$
u_{m}(t)=k p(1+o(1)) \exp (-t), \quad u_{1-m}(t)=x p \lambda^{\alpha}(1+o(1)) \exp (-t) .
$$

На втором шаге - при $t \in[T, 2 T]$ - рассматриваем систему (1) как неоднородную систему обыкновенных дифференциальных уравнений (поскольку функции $F\left(u_{m}(t-T)\right)$ и $F\left(u_{1-m}(t-T)\right)$ при данных $t$ известны). Асимптотика решений на этом временном отрезке имеет вид

где

$$
u_{m}(t)=\lambda\left[g_{k}(t)+o(1)\right], \quad u_{1-m}(t)=x p \lambda^{\alpha}(1+o(1)) \exp (-t)+\frac{\gamma_{1} \lambda^{1-\alpha}}{\ln \lambda}\left[g_{1, k}(t)+o(1)\right],
$$

$$
g_{k}(t)=\int_{T}^{t} \exp (s-t) f(k p \exp (T-s)) d s, \quad g_{1, k}(t)=\int_{T}^{t} \exp (s-t) g_{k}(s) d s .
$$

Заметим, что в зависимости от значения $\alpha$ главным членом асимптотики функции $u_{1-m}(t)$ может быть как первое, так и второе слагаемое. В частности, при $t=2 T$ получаем

$$
\begin{aligned}
u_{m}(2 T) & =\lambda\left[g_{k}(2 T)+o(1)\right], \\
u_{1-m}(2 T) & = \begin{cases}x p \lambda^{1 / 2} \exp (-2 T)(1+o(1)) & \text { при } \alpha=1 / 2, \\
\frac{\gamma_{1} \lambda^{1-\alpha}}{\ln \lambda}\left[g_{1, k}(2 T)+o(1)\right] & \text { при } 0<\alpha<\frac{1}{2} .\end{cases}
\end{aligned}
$$


Предположим, что при $t \in[T, 2 T]$ выполняются следующие условия:

$$
\left\{\begin{array}{l}
g_{k}(t) \neq 0 \text { и } g_{1, k}(t) \neq 0 \text { за исключением конечного числа точек; } \\
\text { если } g_{k}\left(t^{*}\right)=0 \text {, то } g_{k}^{(n)}\left(t^{*}\right) \neq 0 \text { хотя бы для одного } n \text { из множества }\{1,2,3\}, \\
\text { если } g_{1, k}\left(t^{*}\right)=0, \text { то } g_{1, k}^{(n)}\left(t^{*}\right) \neq 0 \text { хотя бы для одного } n \text { из множества }\{1,2,3,4\} .
\end{array}\right.
$$

Пусть выполняется неравенство

$$
g_{k}(2 T) g_{1, k}(2 T)>0 .
$$

Предположим, что главный член асимптотики решения системы (1) на отрезке $t \in[2 T, 3 T]$ совпадает с главным членом асимптотики решения задачи Коши (8), (10). Это условие выполняется, например, при $0<\alpha<1 / 5$ и $\alpha=1 / 2$ для любых функций $f$, удовлетворяющих условиям (3), $(11),(12)$. Из совпадения главных членов асимптотик на отрезке $t \in[2 T, 3 T]$ следует, что решение системы (1) находится вне полосы $[-p, p]$. Следовательно, на следующем шаге $(t \in[3 T, 4 T])$ система (1) имеет вид (8). Данное свойство будет выполняться, пока обе компоненты решения будут вне полосы $[-p, p]$. Обозначим через $t_{*}$ первый момент времени, для которого $t_{*}>2 T$ и $\left|u_{m}\left(t_{*}\right)\right|=p$ или $\left|u_{1-m}\left(t_{*}\right)\right|=p$. Тогда на отрезке $t \in\left[2 T, t_{*}\right]$ выполняются асимптотические равенства

$$
\begin{aligned}
u_{m}(t) & =\lambda\left[g_{k}(2 T)+o(1)\right] \exp (-\tilde{t}), \\
u_{1-m}(t) & = \begin{cases}\lambda^{1 / 2}\left[x p e^{-2 T}+\frac{\gamma_{1} \tilde{t}}{\ln \lambda} g_{k}(2 T)+o\left(\frac{\tilde{t}}{\ln \lambda}\right)\right] \exp (-\tilde{t}), & \text { при } \alpha=\frac{1}{2}, \\
\frac{\gamma_{1} \lambda^{1-\alpha}}{\ln \lambda}\left[g_{1, k}(2 T)+g_{k}(2 T) \tilde{t}+o(1)\right] \exp (-\tilde{t}), & \text { при } 0<\alpha<\frac{1}{2},\end{cases}
\end{aligned}
$$

где $\tilde{t}=t-2 T$. Из формулы (13) при условии (12) очевидно следует, что $\left|u_{1-m}\left(t_{*}\right)\right|=p$ и

$$
t_{*}=(1-\alpha)(1+o(1)) \ln \lambda \quad \text { при } \lambda \rightarrow+\infty .
$$

3. Построение отображения. Из определения точки $t_{*}$ следует, что значения функций $u_{m}$ и $u_{1-m}$ в данной точке можно представить в виде

$$
u_{\bar{m}}\left(t_{*}\right)=\bar{k} p, \quad u_{1-\bar{m}}\left(t_{*}\right)=\bar{x} p \lambda^{\alpha} .
$$

Пусть выполняется условие (12) и неравенство

$$
x p e^{-2 T}+\frac{\gamma_{1}}{2} g_{k}(2 T) \neq 0 .
$$

Тогда, пользуясь асимптотикой решений (13) и формулой (14), находим:

$$
\begin{aligned}
& \bar{m}=1-m, \\
& \bar{k}= \begin{cases}\operatorname{sign}\left(x p e^{-2 T}+\frac{\gamma_{1}}{2} g_{k}(2 T)\right) & \text { при } \alpha=\frac{1}{2}, \\
\operatorname{sign}\left(g_{k}(2 T)\right) & \text { при } 0<\alpha<\frac{1}{2},\end{cases} \\
& \bar{x}= \begin{cases}\frac{g_{k}(2 T)}{\left|x p e^{-2 T}+\frac{\gamma_{1}}{2} g_{k}(2 T)\right|}+o(1) & \text { при } \alpha=\frac{1}{2}, \\
\frac{\operatorname{sign}\left(g_{k}(2 T)\right)}{\gamma_{1}(1-\alpha)}+o(1) & \text { при } 0<\alpha<\frac{1}{2},\end{cases}
\end{aligned}
$$

Из (14) получаем, что при достаточно больших $\lambda>0$ выполняются неравенства

$$
\left|u_{\bar{m}}\left(s+t_{*}\right)\right|>p, \quad\left|u_{1-\bar{m}}\left(s+t_{*}\right)\right|>p \quad \text { при } s \in[-T, 0) .
$$

Таким образом, из (15) и (20) следует, что на отрезке $s \in[-T, 0]$ функции $u_{m}\left(s+t_{*}\right)$ и $u_{1-m}\left(s+t_{*}\right)$ принадлежат множеству $S(\bar{m}, \bar{k}, \bar{x})$. Это означает, что в точке $t_{*}$ мы возвращаемся к исходной (при $t=0)$ ситуации с заменой параметров $m, k, x$ на $\bar{m}, \bar{k}, \bar{x}$ согласно формулам (17), (18) (19). 
Заметим, что компонента $\bar{m}$ не зависит от других компонент отображения, а главные части отображений (18), (19) задают следующие итерационные процессы относительно переменных $k_{n}$, $x_{n}(n=1,2, \ldots)$ :

$$
\left\{\begin{array}{l}
k_{n+1}=\operatorname{sign}\left(g_{k_{n}}(2 T)\right), \\
x_{n+1}=\frac{\operatorname{sign}\left(g_{k_{n}}(2 T)\right)}{\gamma_{1}(1-\alpha)}=: P\left(k_{n}, x_{n}\right)
\end{array}\right.
$$

при $0<\alpha<1 / 2$ и

при $\alpha=1 / 2$.

$$
\left\{\begin{array}{l}
k_{n+1}=\operatorname{sign}\left(x_{n} p e^{-2 T}+\frac{\gamma_{1}}{2} g_{k_{n}}(2 T)\right) \\
x_{n+1}=\frac{g_{k_{n}}(2 T)}{\left|x_{n} p e^{-2 T}+\frac{\gamma_{1}}{2} g_{k_{n}}(2 T)\right|}=: P\left(k_{n}, x_{n}\right)
\end{array}\right.
$$

4. Теорема о соответствии. Для формулировки основного результата введем в рассмотрение следующее отображение

$$
x_{n+1}=P\left(k_{r}^{*}, \ldots P\left(k_{3}^{*},\left(P\left(k_{2}^{*}, P\left(k_{1}^{*}, x_{n}\right)\right)\right)\right) \ldots\right) .
$$

Данное отображение получается как $r$-я итерация компоненты $x$ отображения (21) при $0<\alpha<$ $1 / 2$ (отображения (22) при $\alpha=1 / 2$ соответственно) при зафиксированных значениях компоненты $k\left(k_{i}=k_{i}^{*}\right.$ для всех $\left.i=\overline{1, r}\right)$.

Теорема 1 (Теорема о соответствии). Пусть $0<\alpha<1 / 5(\alpha=1 / 2)$. Пусть выполняются условия (11), (12), (16). Пусть отображение (17), (21) ((17), (22) соответственно) имеет иикл $\left(m_{i}^{*}, k_{i}^{*}, x_{i}^{*}\right)$ периода $r$ такой, что абсолютная величина мультипликатора неподвижной точки $x_{1}^{*}$ отображсения (23) отлична от единицы. Тогда при всех достаточно больших $\lambda>0$ система (1) при условиях (2), (3), (5) имеет иикл периода $T_{*}(\lambda)=(r(1-\alpha)+o(1)) \ln \lambda$ с теми же свойствами устойчивости, что у неподвижной точки $x_{1}^{*}$ отображсения (23).

Доказательство. Доказательство проводится по следующей схеме. Сначала с помощью теоремы о неявной функции доказывается, что если отображение (17), (21) в случае $0<\alpha<1 / 5$ (отображение $(17),(22)$ в случае $\alpha=1 / 2$ соответственно) имеет цикл $\left(m_{i}^{*}, k_{i}^{*}, x_{i}^{*}\right)$ периода $r$, удовлетворяющий условиям теоремы 1 , то отображение, получаемое из условий (15), (20) (главная часть его асимптотики при $\lambda \rightarrow+\infty$ задается формулами $(17),(18),(19))$ имеет цикл $\left(m_{i}^{*}, k_{i}^{*}, \tilde{x}_{i}^{*}\right)$ периода $r$. Для точек $\tilde{x}_{i}^{*}$ данного цикла верны равенства $\tilde{x}_{i}^{*}=x_{i}^{*}+o(1)(i=\overline{1, r})$ при $\lambda \rightarrow+\infty$.

Далее несложно доказывается, что если отображение (15), (20) имеет цикл периода $r$, то при выполнении условий теоремы 1 исходная система (1) при всех достаточно больших значениях $\lambda>0$ имеет релаксационное периодическое решение с периодом $T_{*}(\lambda)=(r(1-\alpha)+o(1)) \ln \lambda$. В самом деле, множество начальных условий $S(m, k, x)$ при любых допустимых фиксированных значениях $m, k, x$ под действием системы (1) «схлопывается» в одно решение. Поэтому если мы в качестве начальной функции возьмем произвольную функцию из множества $S\left(m_{1}^{*}, k_{1}^{*}, \tilde{x}_{1}^{*}\right)$, то через $r$ «шагов» мы получим начальные условия для искомого периодического решения.

В заключение доказывается, что устойчивой неподвижной точке $x_{1}^{*}$ отображения (23) соответствует экспоненциально орбитально устойчивое периодическое решение исходной системы, а неустойчивой неподвижной точке - неустойчивое периодическое решение. Это следует из того, что у изучаемого периодического решения все мультипликаторы, кроме двух, нулевые, один мультипликатор единичный (поскольку решение периодическое), а оставшийся мультипликатор асимптотически близок к мультипликатору неподвижной точки $x_{1}^{*}$ отображения (23).

Подробные выкладки доказательства аналогичной теоремы для близкой к рассматриваемой задачи приведены в работе [8].

Замечание. Условие $\alpha<1 / 5$ продиктовано простотой доказательства того факта, что для любых функций $f$, удовлетворяющих условиям (3), (11), (12), на каждой итерации отображения $(17),(21)$ главный член асимптотики решения системы (1) на отрезке $t \in[2 T, 3 T]$ совпадает 
с главным членом асимптотики решения задачи Коши (8), (10). Если при конкретной функции $f$ при $1 / 5 \leqslant \alpha<1 / 2$ этот факт сохранится, то и при $1 / 5 \leqslant \alpha<1 / 2$ теорема останется верной для рассматриваемой функции $f$.

\section{5. Динамика построенного отображения и устойчивые циклы исходной системы.} Сначала рассмотрим случай $0<\alpha<1 / 2$.

В этом случае построенное отображение задается формулами (17), (21). Динамика отображения зависит от знаков величин $g_{1}(2 T)$ и $g_{-1}(2 T)$.

1. Если обе величины $g_{1}(2 T)$ и $g_{-1}(2 T)$ положительны, то единственный устойчивый цикл $\left(m_{i}, k_{i}, x_{i}\right)$ отображения $(17),(21)$ имеет вид

$$
\left(0,1,\left(\gamma_{1}(1-\alpha)\right)^{-1}\right) \rightarrow\left(1,1,\left(\gamma_{1}(1-\alpha)\right)^{-1}\right) .
$$

2. При $g_{1}(2 T)<0$ и $g_{-1}(2 T)<0$ построенное отображение имеет единственный устойчивый цикл

$$
\left(0,-1,-\left(\gamma_{1}(1-\alpha)\right)^{-1}\right) \rightarrow\left(1,-1,-\left(\gamma_{1}(1-\alpha)\right)^{-1}\right) .
$$

3. Если величина $g_{1}(2 T)$ положительна, а величина $g_{-1}(2 T)$ отрицательна, то устойчивые циклы (24) и (25) сосуществуют.

4. При условиях $g_{1}(2 T)<0$ и $g_{-1}(2 T)>0$ сосуществуют устойчивые циклы

$$
\begin{aligned}
& \left(0,-1,-\left(\gamma_{1}(1-\alpha)\right)^{-1}\right) \rightarrow\left(1,1,\left(\gamma_{1}(1-\alpha)\right)^{-1}\right), \\
& \left(0,1,\left(\gamma_{1}(1-\alpha)\right)^{-1}\right) \rightarrow\left(1,-1,-\left(\gamma_{1}(1-\alpha)\right)^{-1}\right) .
\end{aligned}
$$

Заметим, что все соответствующие экспоненциально орбитально устойчивые циклы исходной системы будут иметь период $T_{*}(\lambda)=(2(1-\alpha)+o(1)) \ln \lambda$ при $\lambda \rightarrow+\infty$. В случаях 1-3 циклы исходной системы $\left(u_{0}(t), u_{1}(t)\right)^{T}$ будут антифазными решениями системы (1) (то есть $u_{0}(t) \equiv u(t)$, $\left.u_{1}(t) \equiv u\left(t+\frac{1}{2} T_{*}(\lambda)\right)\right)$, а в случае 4 не существует такого сдвига $\phi$, что решения системы (1) можно представить в виде $u_{0}(t) \equiv u(t), u_{1}(t) \equiv u(t+\phi)$.

Примеры графиков экспоненциально орбитально устойчивых циклов системы $(1)$ в случае $0<$ $\alpha<1 / 2$ изображены на рис. 1 .

Теперь перейдем к случаю $\alpha=1 / 2$.

В этом случае построенное отображение имеет вид (17), (22). Как и в предыдущем случае, динамика отображения принципиально зависит от знаков величин $g_{1}(2 T)$ и $g_{-1}(2 T)$.

1. При условиях $g_{1}(2 T)>0$ и $g_{-1}(2 T)>0$ единственный устойчивый цикл $\left(m_{i}, k_{i}, x_{i}\right)$ отображения (17), (22) имеет вид

$$
\left(0,1, x_{*}\right) \rightarrow\left(1,1, x_{*}\right) .
$$

Здесь $x_{*}$ вычисляется по формуле

$$
x_{*}=\frac{-\frac{\gamma_{1}}{2} g_{1}(2 T)+\sqrt{\frac{\gamma_{1}^{2}}{4} g_{1}^{2}(2 T)+4 p e^{-2 T} g_{1}(2 T)}}{2 p e^{-2 T}} .
$$

2. При выполнении неравенств $g_{1}(2 T)<0$ и $g_{-1}(2 T)<0$ устойчивый цикл построенного отображения имеет вид

$$
\left(0,-1, x^{*}\right) \rightarrow\left(1,-1, x^{*}\right) .
$$

Здесь константа $x^{*}$ находится по формуле

$$
x^{*}=\frac{-\frac{\gamma_{1}}{2} g_{-1}(2 T)-\sqrt{\frac{\gamma_{1}^{2}}{4} g_{-1}^{2}(2 T)-4 p e^{-2 T} g_{-1}(2 T)}}{2 p e^{-2 T}} .
$$

3. Если величина $g_{1}(2 T)$ положительна, а величина $g_{-1}(2 T)$ отрицательна, то устойчивые циклы (28) и (29) сосуществуют.

4. Если же величина $g_{1}(2 T)$ отрицательна, а величина $g_{-1}(2 T)$ положительна, то при различных функциях $f(u)$ у отображения $(17),(22)$ могут существовать устойчивые циклы различных периодов. 
a)

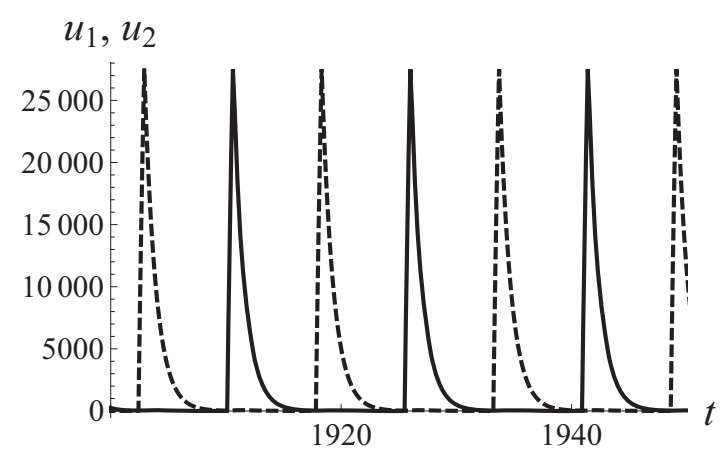

c)

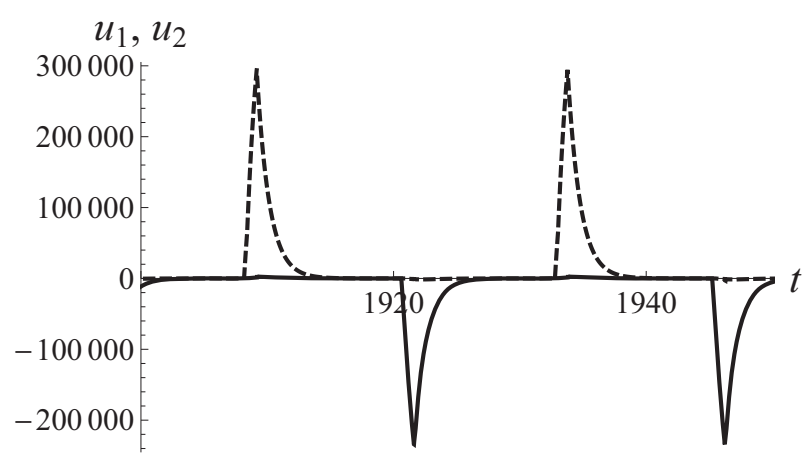

b)

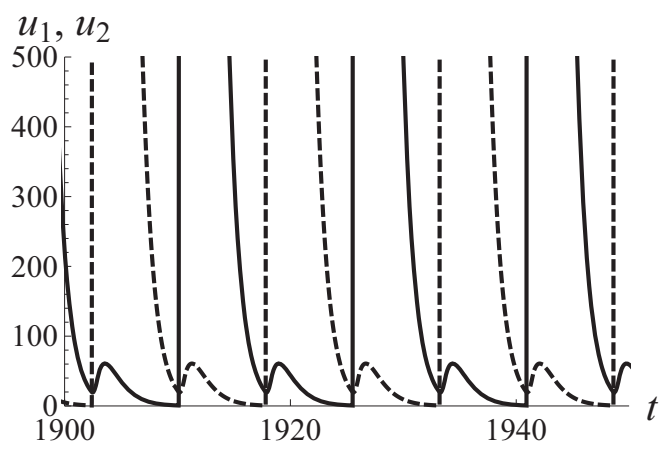

d)

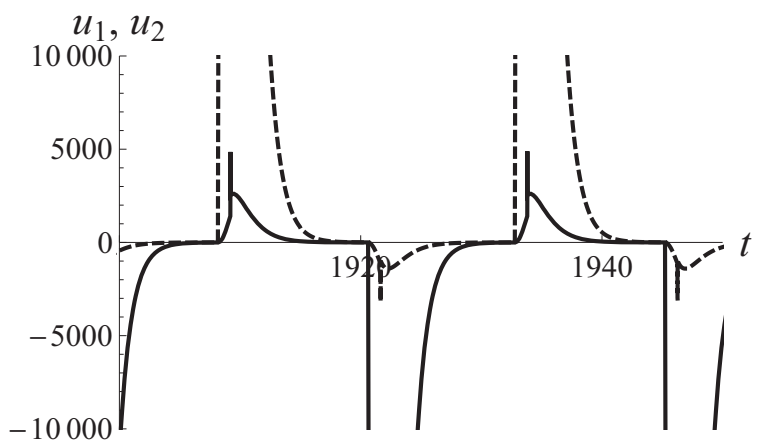

Рис. 1. (а) Цикл системы (1), соответствующий циклу (24) отображения (17), (21) (случай $g_{1}(2 T)>0$ и $g_{-1}(2 T)>0$; значения параметров: $\left.\lambda=10000, T=0,5, \gamma_{1}=0,1, \alpha=0,1\right)$. (b) Увеличенный фрагмент рис. 1(а). (с) Цикл системы (1), соответствующий циклу (26) отображения (17), $(21)$ (случай $g_{1}(2 T)<0$ и $g_{-1}(2 T)>0$; значения параметров: $\lambda=10000, T=1, \gamma_{1}=0,25$, $\alpha=0,09)$. (d) Увеличенный фрагмент рис. 1(с).

Здесь, как и при $0<\alpha<1 / 2$, в первых трех случаях соответствующие решения исходной системы (1) будут антифазными периодическими решениями. А в четвертом случае (в зависимости от конкретной функции $f$ ) у исходной системы возможны как антифазные циклы, так и циклы, не представимые в виде $u_{0}(t) \equiv u(t), u_{1}(t) \equiv u(t+\phi)$, где $\phi$ - некоторая константа.

На рис. 2 изображены примеры графиков экспоненциально орбитально устойчивых циклов системы (1) в случае $\alpha=1 / 2$.

Заметим, что решение, изображенное на рис. 1.(а), можно трактовать как импульсный режим, а решение, изображенное на рис. 2(a), можно интерпретировать как пачку импульсов.

6. Заключение. В данной работе исследована нелокальная динамика двух слабо связанных осцилляторов с нелинейной финитной запаздывающей обратной связью. Благодаря тому, что перед функцией обратной связи стоит множитель, являющийся достаточно большим, для аналитического исследования задачи удается применить специальный асимптотический метод. В результате задача о нелокальной динамике исходной бесконечномерной системы с большим параметром сведена к изучению динамики построенного трехмерного отображения без больших и малых параметров: грубые циклы данного отображения соответствуют релаксационным периодическим решениям исходной системы. Найдены грубые устойчивые циклы построенного отображения и приведены графики соответствующих им экспоненциально орбитально устойчивых релаксационных периодических решений исходной системы. Полученные экспоненциально орбитально 
a)

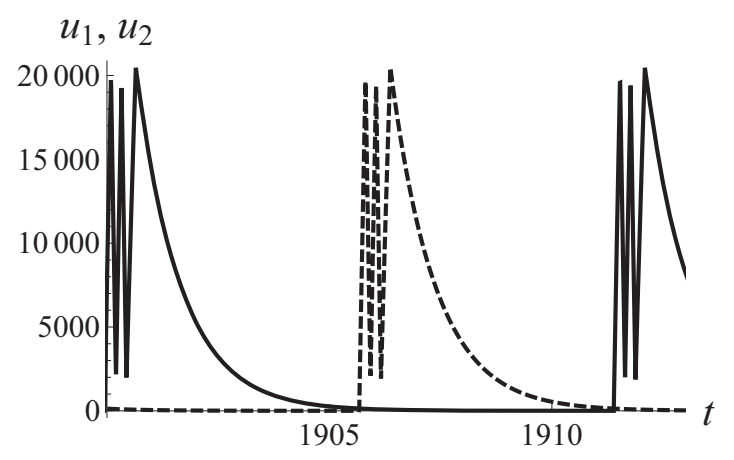

c)

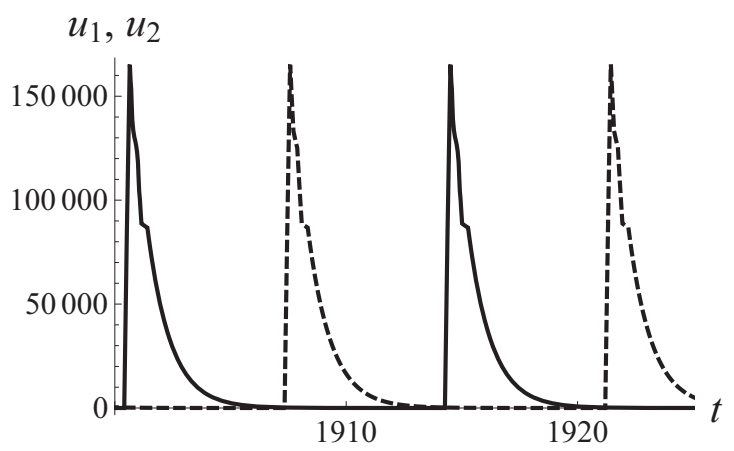

b)

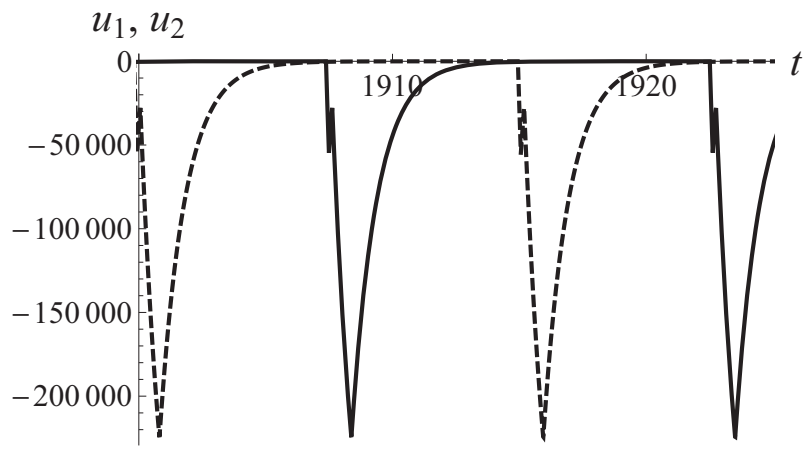

d)

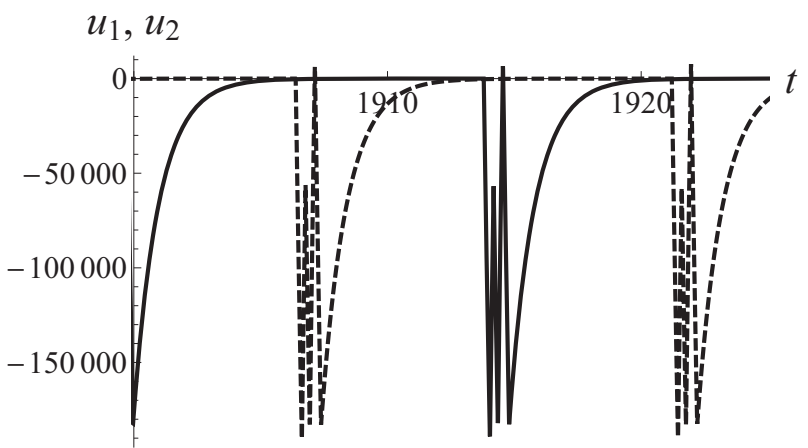

Рис. 2. (а) Цикл системы (1), соответствующий циклу (28) отображения (17), (22) (случай $g_{1}(2 T)>0$ и $g_{-1}(2 T)>0$; значения параметров: $\left.\lambda=10000, T=0,7, \gamma_{1}=0,08\right)$. (b) Цикл системы (1), соответствующий циклу (29) отображения (17), (22) (случай $g_{1}(2 T)<0$ и $g_{-1}(2 T)<0$; значения параметров: $\left.\lambda=10000, T=1, \gamma_{1}=0,1\right)$. (c), (d) сосуществующие циклы системы $(1)$ (случай $g_{1}(2 T)>0$ и $g_{-1}(2 T)<0$. Рис. (c) соответствует циклу $(28),($ d) соответствует циклу $(29)$. Значения параметров: $\left.\lambda=10000, T=1, \gamma_{1}=0,1\right)$.

устойчивые циклы могут быть как антифазными решениями, так и решениями, не представимыми в виде $u_{0}(t) \equiv u(t), u_{1}(t) \equiv u(t+\phi)$, где $\phi$ - некоторая константа. Приведены графики решений, которые можно трактовать как импульсные режимы и как пачки импульсов.

\section{СПИСОК ЛИТЕРАТУРЫ}

1. Глызин С. Д., Колесов А. Ю., Розов Н. Х. Дискретные автоволны в нейронных системах// Ж. вычисл. мат. мат. физ. - 2012. - 52, № 5. - С. 840-858.

2. Глызин С. Д., Колесов А. Ю., Розов Н. Х. Моделирование эффекта взрыва в нейронных системах// Мат. заметки. - 2013. - 93, № 5. - С. 684-701.

3. Дмитриев А. С., Кислов В. Я. Стохастические колебания в радиофизике и электронике. - М.: Наука, 1989.

4. Кащенко С. А. Исследование методами большого параметра системы нелинейных дифференциальноразностных уравнений, моделирующих задачу хищник-жертва// Докл. АН СССР. - 1982. - 266, № 4 . - C. $792-795$.

5. Кащенко С. А., Майоров В. В. Модели волновой памяти. - М.: Либроком, 2009.

6. Grigorieva E. V., Kaschenko S. A. Asymptotic Representation of Relaxation Oscillations in Lasers. Springer-Verlag, 2017.

7. Kashchenko A. A. A family of non-rough cycles in a system of two coupled delayed generators// Automat. Control Comput. Sci. - 2017. — 51, № 7. - P. 753-756. 
8. Kashchenko A. A. Multistability in a system of two coupled oscillators with delayed feedback// J. Differ. Equations. - 2019. - 266, № 1. - P. 562-579.

9. Kashchenko A. A., Kaschenko S. A. Asymptotic behavior of the solutions of a system of two weakly coupled relaxation oscillators with delayed feedback// Radiophys. Quant. Electronics. — 2019. — 61, № 8-9. P. 633-639.

10. Kilias T., Kelber K., Mogel A., and Schwarz W. Electronic chaos generators-design and applications// Int. J. Electronics. — 1995. — 79, № 6. - P. 737-753.

11. Preobrazhenskaia $M . M$. The impulse-refractive mode in a neural network with ring synaptic interaction// Automat. Control Comput. Sci. — 2018. - 52, № 7. - P. 777-789.

12. Rabinovich M. I., Varona P., Selverston A. I., Abarbanel H. D. I. Dynamical principles in neuroscience// Rev. Mod. Phys. — 2006. - 78, № 4. - P. 1213-1265.

Кащенко Александра Андреевна

Ярославский государственный университет им. П. Г. Демидова

E-mail: sa-ahr@yandex.ru 